\title{
On some nonlocal elliptic systems with multiple parameters
}

\section{Sur certains systèmes elliptiques non locaux à paramètres multiples}

\author{
Sh. Shahriyari ${ }^{1}$, H. Zahmatkesh ${ }^{1, *}$, S. Shakeri ${ }^{2}$, and A. Hadjian ${ }^{3}$ \\ ${ }^{1}$ Department of Mathematics, Gorgan Branch, Islamic Azad University, Gorgan, Iran \\ shahrozshahriyari@yahoo.com, zahmatkesh_h@yahoo.com \\ ${ }^{2}$ Department of Mathematics, Ayatollah Amoli Branch, Islamic Azad University, Amol, Iran \\ s.shakeri@iauamol.ac.ir \\ ${ }^{3}$ Department of Mathematics, Faculty of Basic Sciences, University of Bojnord, P.O. Box 1339, Bojnord 94531, Iran \\ hadjian83@gmail.com, a.hadjian@ub.ac.ir
}

ABSTRACT. In this paper, using sub-supersolution argument, we prove some existence results on positive solution for a class of nonlocal elliptic systems with multiple parameters in bounded domains.

2010 Mathematics Subject Classifications: 35D30, 35J60.

KEYWORDS. Kirchhoff type problems; Semipositone; Positive solution; Sub- and supersolutions.

\section{Introduction}

In this paper, we are interested in a class of nonlinear elliptic systems of the form

$$
\left\{\begin{array}{lc}
-M_{1}\left(\int_{\Omega}|\nabla u|^{p} d x\right) \Delta_{p} u=\lambda_{1} a(x) f(v)+\mu_{1} c(x) h(u) & \text { in } \Omega, \\
-M_{2}\left(\int_{\Omega}|\nabla v|^{p} d x\right) \Delta_{p} v=\lambda_{2} b(x) g(u)+\mu_{2} d(x) \tau(v) & \text { in } \Omega, \\
u=v=0 & \text { on } \partial \Omega,
\end{array}\right.
$$

where $\Omega \subset \mathbb{R}^{N}(N \geq 3)$ is a bounded domain with smooth boundary $\partial \Omega, M_{i}: \mathbb{R}^{+} \rightarrow \mathbb{R}^{+}, i=1,2$, are continuous functions, $1<p<N, \lambda_{1}, \lambda_{2}, \mu_{1}$ and $\mu_{2}$ are positive parameters and $a, b, c, d$ are $C^{1}$ sign-changing weight functions, that maybe negative near the boundary. $f, g, h, \tau$ are $C^{1}$ non-decreasing non-negative functions on $(0, \infty)$.

Since the first equation in (1.1) contains an integral over $\Omega$, it is no longer a pointwise identity; therefore it is often called nonlocal problem. This problem models several physical and biological systems, where $u$ describes a process which depends on the average of itself, such as the population density, see [5]. Moreover, problem (1.1) is related to the stationary version of the Kirchhoff equation

$$
\rho \frac{\partial^{2} u}{\partial t^{2}}-\left(\frac{P_{0}}{h}+\frac{E}{2 L} \int_{0}^{L}\left|\frac{\partial u}{\partial x}\right|^{2} d x\right) \frac{\partial^{2} u}{\partial x^{2}}=0,
$$

presented by Kirchhoff in 1883, see [11]. This equation is an extension of the classical d'Alembert's wave equation by considering the effects of the changes in the length of the string during the vibrations. The parameters in (1.2) have the following meanings: $L$ is the length of the string, $h$ is the area of the cross-section, $E$ is the Young modulus of the material, $\rho$ is the mass density, and $P_{0}$ is the initial tension.

* Corresponding author. 
In recent years, problems involving Kirchhoff type operators have been studied in many papers, we refer to [3, 4, 6, 7, 12, 14, 15], in which the authors have used different methods to get the existence of solutions for (1.2). In the papers [13, 16], Zhang and Perera studied the existence of nontrivial solutions and sign-changing solutions for (1.1).

In this paper, we are interested in finding positive solutions for system (1.1) via sub-supersolution method. Our paper is motivated by the recent results in [1, 2, 3, 4, 10]. In the paper [4], the authors studied the existence of a positive solution for the nonlocal problem of the form

$$
\left\{\begin{array}{lc}
-M\left(\int_{\Omega}|\nabla u|^{2} d x\right) \Delta u=|u|^{p-1} u+\lambda f(x) & \text { in } \Omega, \\
u=0 & \text { on } \partial \Omega,
\end{array}\right.
$$

where $\Omega$ is a smooth bounded domain in $\mathbb{R}^{N}, N \geq 3$ and $p>1$, i.e. the nonlinear term is superlinear at infinity, $f$ is a sign-changing function. Using the sub-supersolution method combining a comparison principle introduced in [3], the authors established the existence of a positive solution for (1.3) when the parameter $\lambda>0$ is small enough. It was also prove in [4] that problem (1.3) has no positive when $\lambda$ large enough. In [2] Afrouzi et al. investigated the existence and nonexistence of positive solution to the following elliptic system:

$$
\begin{cases}-M_{1}\left(\int_{\Omega}|\nabla u|^{2} d x\right) \Delta_{u}=\lambda f(v) & \text { in } \Omega \\ -M_{2}\left(\int_{\Omega}|\nabla v|^{2} d x\right) \Delta_{v}=\lambda g(u) & \text { in } \Omega \\ u=v=0 & \text { on } \partial \Omega .\end{cases}
$$

In the present paper, we consider system (1.1) in the case when the nonlinearities are sublinear at infinity. We are inspired by the ideas in the interesting paper [10], in which the authors considered (1.1) in the case $M_{1}(t)=M_{2}(t) \equiv 1$. Here we focus on further extending the study in [2] to the system (1.1) which features multiple parameters, weight functions and stronger coupling. More precisely, under suitable conditions on $f, g, h, \tau$ we shall show that system (1.1) has a positive solution for $\lambda_{i}, \mu_{i}, i=1,2$ sufficiently large.

Proposition 1.1. Assume that $M: \mathbb{R}^{+} \rightarrow \mathbb{R}^{+}$is a continuous and increasing function satisfying

$$
M(t) \geq m_{0} \quad \text { for all } t \in \mathbb{R}^{+},
$$

where $m_{0}$ is a positive constant. Assume that $u, v$ are two non-negative functions such that

$$
\left\{\begin{array}{lc}
-M\left(\int_{\Omega}|\nabla u|^{p} d x\right) \Delta_{p} u \geq-M\left(\int_{\Omega}|\nabla v|^{p} d x\right) \Delta_{p} v \quad \text { in } \Omega \\
u=v=0 & \text { on } \partial \Omega .
\end{array}\right.
$$

Then $u \geq v$ a.e. in $\Omega$.

Proof. Our proof is based on the arguments presented in [8, 9]. Define the functional $\Phi: W_{0}^{1, p}(\Omega) \rightarrow \mathbb{R}$ by the formula

$$
\Phi(u):=\frac{1}{p} \widehat{M}\left(\int_{\Omega}|\nabla u|^{p} d x\right), \quad u \in W_{0}^{1, p}(\Omega) .
$$

It is obvious that the functional $\Phi$ is a continuously Gâteaux differentiable whose Gâteaux derivative at the point $u \in W_{0}^{1, p}(\Omega)$ is the functional $\Phi^{\prime} \in W_{0}^{-1, p}(\Omega)$, given by

$$
\Phi^{\prime}(u)(\varphi)=M\left(\int_{\Omega}|\nabla u|^{p} d x\right) \int_{\Omega}|\nabla u|^{p-2} \nabla u \cdot \nabla \varphi d x, \quad \varphi \in W_{0}^{1, p}(\Omega) .
$$


It is obvious that $\Phi^{\prime}$ is continuous and bounded since the function $M$ is continuous. We will show that $\Phi^{\prime}$ is strictly monotone in $W_{0}^{1, p}(\Omega)$. Indeed, for any $u, v \in W_{0}^{1, p}(\Omega), u \neq v$, without loss of generality, we may assume that

$$
\int_{\Omega}|\nabla u|^{p} d x \geq \int_{\Omega}|\nabla v|^{p} d x
$$

(otherwise, changing the role of $u$ and $v$ in the following proof). Therefore, we have

$$
M\left(\int_{\Omega}|\nabla u|^{p} d x\right) \geq M\left(\int_{\Omega}|\nabla v|^{p} d x\right)
$$

since $M(t)$ is a monotone function. Using Cauchy's inequality, we have

$$
\nabla u \cdot \nabla v \leq|\nabla u||\nabla v| \leq \frac{1}{2}\left(|\nabla u|^{2}+|\nabla v|^{2}\right) .
$$

Using (1.8) we get

$$
\int_{\Omega}|\nabla u|^{p} d x-\int_{\Omega}|\nabla u|^{p-2} \nabla u \cdot \nabla v d x \geq \frac{1}{2} \int_{\Omega}|\nabla u|^{p-2}\left(|\nabla u|^{2}-|\nabla v|^{2}\right) d x,
$$

and

$$
\int_{\Omega}|\nabla v|^{p} d x-\int_{\Omega}|\nabla v|^{p-2} \nabla v \cdot \nabla u d x \geq \frac{1}{2} \int_{\Omega}|\nabla v|^{p-2}\left(|\nabla v|^{2}-|\nabla u|^{2}\right) d x .
$$

If $|\nabla u| \geq|\nabla v|$, using (1.7)-(1.10) we have

$$
\begin{aligned}
I_{1}:= & \Phi^{\prime}(u)(u)-\Phi^{\prime}(u)(v)-\Phi^{\prime}(v)(u)+\Phi^{\prime}(v)(v) \\
= & M\left(\int_{\Omega}|\nabla u|^{p} d x\right)\left(\int_{\Omega}|\nabla u|^{p} d x-\int_{\Omega}|\nabla u|^{p-2} \nabla u \cdot \nabla v d x\right) \\
& -M\left(\int_{\Omega}|\nabla v|^{p} d x\right)\left(\int_{\Omega}|\nabla v|^{p-2} \nabla v \cdot \nabla u d x-\int_{\Omega}|\nabla v|^{p} d x\right) \\
\geq & \frac{1}{2} M\left(\int_{\Omega}|\nabla u|^{p} d x\right) \int_{\Omega}|\nabla u|^{p-2}\left(|\nabla u|^{2}-|\nabla v|^{2}\right) d x \\
& -\frac{1}{2} M\left(\int_{\Omega}|\nabla v|^{p} d x\right) \int_{\Omega}|\nabla u|^{p-2}\left(|\nabla u|^{2}-|\nabla v|^{2}\right) d x \\
= & \frac{1}{2} M\left(\int_{\Omega}|\nabla v|^{p} d x\right) \int_{\Omega}\left(|\nabla u|^{p-2}-|\nabla v|^{p-2}\right)\left(|\nabla u|^{2}-|\nabla v|^{2}\right) d x \\
\geq & \frac{M_{0}}{2} \int_{\Omega}\left(|\nabla u|^{p-2}-|\nabla v|^{p-2}\right)\left(|\nabla u|^{2}-|\nabla v|^{2}\right) d x .
\end{aligned}
$$


If $|\nabla v| \geq|\nabla u|$, changing the role of $u$ and $v$ in (1.7)-(1.11) we have

$$
\begin{aligned}
I_{2}:= & \Phi^{\prime}(v)(v)-\Phi^{\prime}(v)(u)-\Phi^{\prime}(u)(v)+\Phi^{\prime}(u)(u) \\
= & M\left(\int_{\Omega}|\nabla v|^{p} d x\right)\left(\int_{\Omega}|\nabla v|^{p} d x-\int_{\Omega}|\nabla v|^{p-2} \nabla v \cdot \nabla u d x\right) \\
& -M\left(\int_{\Omega}|\nabla u|^{p} d x\right)\left(\int_{\Omega}|\nabla u|^{p-2} \nabla u \cdot \nabla v d x-\int_{\Omega}|\nabla u|^{p} d x\right) \\
\geq & \frac{1}{2} M\left(\int_{\Omega}|\nabla v|^{p} d x\right) \int_{\Omega}|\nabla v|^{p-2}\left(|\nabla v|^{2}-|\nabla u|^{2}\right) d x \\
& -\frac{1}{2} M\left(\int_{\Omega}|\nabla u|^{p} d x\right) \int_{\Omega}|\nabla u|^{p-2}\left(|\nabla v|^{2}-|\nabla u|^{2}\right) d x \\
= & \frac{1}{2} M\left(\int_{\Omega}|\nabla v|^{p} d x\right) \int_{\Omega}\left(|\nabla v|^{p-2}-|\nabla u|^{p-2}\right)\left(|\nabla v|^{2}-|\nabla u|^{2}\right) d x \\
\geq & \frac{M_{0}}{2} \int_{\Omega}\left(|\nabla v|^{p-2}-|\nabla u|^{p-2}\right)\left(|\nabla v|^{2}-|\nabla u|^{2}\right) d x .
\end{aligned}
$$

From (1.11) and (1.12) we have

$$
\left(\Phi^{\prime}(u)-\Phi^{\prime}(v)\right)(u-v)=I_{1}=I_{2} \geq 0, \quad \forall u, v \in W_{0}^{1, p}(\Omega) .
$$

Moreover, if $u \neq v$ and $\left(\Phi^{\prime}(u)-\Phi^{\prime}(v)\right)(u-v)=0$, then we have

$$
\int_{\Omega}\left(|\nabla u|^{p-2}-|\nabla v|^{p-2}\right)\left(|\nabla u|^{2}-|\nabla v|^{2}\right) d x=0
$$

so $|\nabla u|=|\nabla v|$ in $\Omega$. Thus, we deduce that

$$
\begin{aligned}
\left(\Phi^{\prime}(u)-\Phi^{\prime}(v)\right)(u-v) & =\Phi^{\prime}(u)(u-v)-\Phi^{\prime}(v)(u-v) \\
& =M\left(\int_{\Omega}|\nabla u|^{p} d x\right) \int_{\Omega}|\nabla u|^{p-2}|\nabla u-\nabla v|^{2} d x \\
& =0,
\end{aligned}
$$

i.e., $u-v$ is a constant. In view of $u=v=0$ on $\partial \Omega$ we have $u \equiv v$ which is contrary with $u \neq v$. Therefore $\left(\Phi^{\prime}(u)-\Phi^{\prime}(v)\right)(u-v)>0$ and $\Phi^{\prime}$ is strictly monotone in $W_{0}^{1, p}(\Omega)$.

Let $u, v$ be two functions such that (1.6) is verified. Taking $\varphi=(u-v)^{+}$, the positive part of $u-v$, as a test function of (1.6), we have 


$$
\begin{aligned}
\left(\Phi^{\prime}(u)-\Phi^{\prime}(v)\right)(\varphi)= & M\left(\int_{\Omega}|\nabla u|^{p} d x\right) \int_{\Omega}|\nabla u|^{p-2} \nabla u \cdot \nabla \varphi d x \\
& -M\left(\int_{\Omega}|\nabla v|^{p} d x\right) \int_{\Omega}|\nabla v|^{p-2} \nabla v \cdot \nabla \varphi d x \\
\leq & 0 .
\end{aligned}
$$

Relations (1.14) and (1.15) mean that $u \leq v$.

\section{Existence result}

In this section, we shall state and prove the main result of this paper. Let $\lambda_{p}$ be the first eigenvalue of $-\Delta_{p}$ with Dirichlet boundary conditions and $\phi$ the corresponding positive eigenfunction with $\|\phi\|_{\infty}=1$. Then there exist positive constants $m_{0}, \delta, r$ such that $|\nabla \phi|^{p}-\lambda_{p} \phi^{p} \geq m_{0}$ on $\bar{\Omega}_{\delta}:=$ $\{x \in \Omega: d(x, \partial \Omega) \leq \delta\}$ and $\phi \geq r$ on $\Omega \backslash \bar{\Omega}_{\delta}$.

We assume that the weight functions $a, b, c, d$ take negative values in $\bar{\Omega}_{\delta}$ but require $a, b, c, d$ to be strictly positive in $\Omega \backslash \Omega_{\delta}$. To be precise we assume that there exist positive constants $a_{0}, b_{0}, c_{0}, d_{0}$ and $a_{1}$, $b_{1}, c_{1}, d_{1}$ such that $a(x) \geq-a_{0}, b(x) \geq-b_{0}, c(x) \geq-c_{0}, d(x) \geq-d_{0}$ on $\Omega_{\delta}$ and $a(x) \geq a_{1}, b(x) \geq b_{1}$, $c(x) \geq c_{1}, d(x) \geq d_{1}$ on $\Omega \backslash \Omega_{\delta}$.

In what follows, if $f_{1}, f_{2}, g_{1}, g_{2}$ are real-valued functions, we write $\left(f_{1}, f_{2}\right) \leq\left(g_{1}, g_{2}\right)$ if and only if $f_{1} \leq g_{1}$ and $f_{2} \leq g_{2}$.

In such a case, we write $(u, v) \in\left[\left(f_{1}, f_{2}\right),\left(g_{1}, g_{2}\right)\right]$ if and only if $f_{1} \leq u \leq g_{1}$ and $f_{2} \leq v \leq g_{2}$.

Let us assume the following assumptions:

$\left(H_{1}\right)$ Assume that $M_{1}, M_{2}: \mathbb{R}^{+} \rightarrow \mathbb{R}^{+}$are two continuous and increasing functions and there exists $m_{1}, m_{2}>0$ such that

$$
M_{1}(t) \geq m_{1}, \quad M_{2}(t) \geq m_{2} \quad \text { for all } t \in \mathbb{R}^{+} .
$$

$\left(H_{2}\right) f, g:[0,+\infty) \rightarrow \mathbb{R}$ are $C^{1}$ increasing functions such that

$$
\lim _{t \rightarrow+\infty} f(t)=\lim _{t \rightarrow+\infty} g(t)=+\infty
$$

$\left(H_{3}\right)$ It holds that

$$
\lim _{t \rightarrow+\infty} \frac{f\left(K\left(g(t)^{\frac{1}{p-1}}\right)\right)}{t^{p-1}}=0
$$

for every constant $K>0$.

$\left(H_{4}\right) h, \tau \in C^{1}([0, \infty))$ are non-negative, non-decreasing functions such that

$$
\lim _{u \rightarrow+\infty} \frac{h(u)}{u^{p-1}}=0, \quad \lim _{u \rightarrow+\infty} \frac{\tau(u)}{u^{p-1}}=0, \quad \lim _{u \rightarrow+\infty} h(u)=\lim _{u \rightarrow+\infty} \tau(u)=+\infty .
$$


$\left(H_{5}\right)$ There exists $\gamma, s>0$ such that

$$
\begin{aligned}
& \min \left\{\left(\frac{a_{0} f\left((\gamma+s)^{\frac{1}{p-1}}\right)}{\gamma}, \frac{c_{0} h\left((\gamma+s)^{\frac{1}{p-1}}\right)}{s}\right),\left(\frac{b_{0} g\left((\gamma+s)^{\frac{1}{p-1}}\right)}{\gamma}, \frac{d_{0} \tau\left((\gamma+s)^{\frac{1}{p-1}}\right)}{s}\right)\right\} \\
& <\min \left\{\left(\frac{a_{1} f\left((\gamma+s)^{\frac{1}{p-1}} \frac{p-1}{p} r^{\frac{p}{p-1}}\right)}{s \lambda_{p}}, \frac{c_{1} h\left((\gamma+s)^{\frac{1}{p-1}} \frac{p-1}{p} r^{\frac{p}{p-1}}\right)}{\gamma \lambda_{p}}\right),\right. \\
& \left.\left(\frac{b_{1} g\left((\gamma+s)^{\frac{1}{p-1}} \frac{p-1}{p} r^{\frac{p}{p-1}}\right)}{s \lambda_{p}}, \frac{d_{1} h\left((\gamma+s)^{\frac{1}{p-1}} \frac{p-1}{p} r^{\frac{p}{p-1}}\right)}{\gamma \lambda_{p}}\right)\right\} \\
& \left(\frac{1}{\|b\|_{\infty}}, \frac{1}{\|d\|_{\infty}}\right)< \\
& \min \left\{\left(\frac{a_{1} f\left((\gamma+s)^{\frac{1}{p-1} \frac{p-1}{p} r^{\frac{p}{p-1}}}\right)}{s \lambda_{p}}, \frac{c_{1} h\left((\gamma+s)^{\frac{1}{p-1}} \frac{p-1}{p} r^{\frac{p}{p-1}}\right)}{\gamma \lambda_{p}}\right),\right. \\
& \left.\left(\frac{b_{1} g\left((\gamma+s)^{\frac{1}{p-1}} \frac{p-1}{p} r^{\frac{p}{p-1}}\right)}{s \lambda_{p}}, \frac{d_{1} h\left((\gamma+s)^{\frac{1}{p-1}} \frac{p-1}{p} r^{\frac{p}{p-1}}\right)}{\gamma \lambda_{p}}\right)\right\} \text {. }
\end{aligned}
$$

It should be noticed that the nonlinearities $\alpha_{i} t^{q_{i}}-\beta_{i}$, where $t \in \mathbb{R}^{+}, \alpha_{i}, \beta_{i}>0, q_{i} \in(0, p-1)$, $1 \leq i \leq 4$, for the functions $f, g, h$ and $\tau$, satisfy the condition $\left(H_{5}\right)$.

Our main result in this paper is given by the following theorem.

Theorem 2.1. Assume that the conditions $\left(H_{1}\right)-\left(H_{5}\right)$ hold. Then there exists $[A, B]$ such that problem (1.1) has a positive solution $(u, v)$ for every $\left(\lambda_{i}, \mu_{i}\right) \in[A, B], i=1,2$.

Proof. Define $f(t)=0$ and $g(t)=0$ for all $t<0$. We shall establish Theorem 2.1 by constructing a positive weak subsolution $(\underline{u}, \underline{v}) \in W_{0}^{1, p}\left(\Omega, \mathbb{R}^{2}\right)$ and a positive supersolution $(\bar{u}, \bar{v}) \in W_{0}^{1, p}\left(\Omega, \mathbb{R}^{2}\right)$ of problem (1.1) such that $\underline{u} \leq \bar{u}$ and $\underline{v} \leq \bar{v}$ in $\Omega$. That is, $\underline{u}, \bar{u}, \underline{v}, \bar{v}$ satisfies

$$
\begin{aligned}
& M_{1}\left(\int_{\Omega}|\nabla \underline{u}|^{p} d x\right) \int_{\Omega}|\nabla \underline{u}|^{p-2} \nabla \underline{u} \cdot \nabla \varphi d x \leq \int_{\Omega}\left[\lambda_{1} a(x) f(\underline{v})+\mu_{1} c(x) h(\underline{u})\right] \varphi d x \\
& M_{2}\left(\int_{\Omega}|\nabla \underline{v}|^{p} d x\right) \int_{\Omega}|\nabla \underline{v}|^{p-2} \nabla \underline{v} \cdot \nabla \varphi d x \leq \int_{\Omega}\left[\lambda_{2} b(x) g(\underline{v})+\mu_{2} d(x) \tau(\underline{u})\right] \varphi d x
\end{aligned}
$$

and

$$
\begin{aligned}
& M_{1}\left(\int_{\Omega}|\nabla \bar{u}|^{p} d x\right) \int_{\Omega}|\nabla \bar{u}|^{p-2} \nabla \underline{u} \cdot \nabla \varphi d x \geq \int_{\Omega}\left[\lambda_{1} a(x) f(\bar{v})+\mu_{1} c(x) h(\bar{u})\right] \varphi d x \\
& M_{2}\left(\int_{\Omega}|\nabla \bar{v}|^{p} d x\right) \int_{\Omega}|\nabla \bar{v}|^{p-2} \nabla \underline{v} \cdot \nabla \varphi d x \geq \int_{\Omega}\left[\lambda_{2} b(x) g(\bar{v})+\mu_{2} d(x) \tau(\bar{u})\right] \varphi d x
\end{aligned}
$$

for all $\varphi \in W_{0}^{1, p}(\Omega)$ with $\varphi \geq 0$.

We define

$$
A=\max \left\{\left(\frac{s \lambda_{p}}{b_{1} g\left((\gamma+s)^{\frac{1}{p-1}} \frac{p-1}{p} r^{\frac{p}{p-1}}\right)}, \frac{\gamma \lambda_{p}}{d_{1} h\left((\gamma+s)^{\left.\frac{1}{p-1} \frac{p-1}{p} r^{\frac{p}{p-1}}\right)}\right.}\right)\right.
$$




$$
\begin{aligned}
& \left.\left(\frac{s \lambda_{p}}{a_{1} f\left((\gamma+s)^{\frac{1}{p-1}} \frac{p-1}{p} r^{\frac{p}{p-1}}\right)}, \frac{\gamma \lambda_{p}}{c_{1} h\left((\gamma+s)^{\frac{1}{p-1}} \frac{p-1}{p} r^{\frac{p}{p-1}}\right)}\right)\right\}, \\
& B=\min \left\{\left(\frac{s}{b_{0} g\left((\gamma+s)^{\frac{1}{p-1}}\right)}, \frac{\gamma}{d_{0} h\left((\gamma+s)^{\frac{1}{p-1}}\right)}\right),\right. \\
& \left.\left(\frac{s}{a_{0} f\left((\gamma+s)^{\frac{1}{p-1}}\right)}, \frac{\gamma}{c_{0} h\left((\gamma+s)^{\frac{1}{p-1}}\right)}\right),\left(\frac{1}{\|b\|_{\infty}}, \frac{1}{\|d\|_{\infty}}\right)\right\} \text {. }
\end{aligned}
$$

Let us define

$$
\underline{u}=(\gamma+s)^{\frac{1}{p-1}} \frac{p-1}{p}\left(\frac{1}{m_{1} m_{0}}\right)^{\frac{1}{p-1}} \phi^{\frac{p}{p-1}}, \quad \underline{v}=(\gamma+s)^{\frac{1}{p-1}} \frac{p-1}{p}\left(\frac{1}{m_{2} m_{0}}\right)^{\frac{1}{p-1}} \phi^{\frac{p}{p-1}},
$$

where $m_{1}, m_{2}$ are given by the condition $\left(H_{1}\right)$. We shall verify that $(\underline{u}, \underline{v})$ is a subsolution of problem (1.1) for $\left(\lambda_{i}, \mu_{i}\right) \in[A, B], i=1,2$. Indeed, let $\varphi \in W_{0}^{1, p}(\Omega)$ with $\varphi \geq 0$ in $\Omega$. By $\left(H_{1}\right)$, a simple calculation shows that

$$
\begin{aligned}
& M_{1}\left(\int_{\Omega}|\nabla \underline{u}|^{p} d x\right) \int_{\Omega}|\nabla \underline{u}|^{p-2} \nabla \underline{u} \cdot \nabla \varphi d x \\
&=M_{1}\left(\int_{\Omega}|\nabla \underline{u}|^{p} d x\right) \frac{(\gamma+s)}{m_{1} m_{0}} \int_{\Omega} \phi|\nabla \phi|^{p-2} \nabla \phi \cdot \nabla \varphi d x \\
&=\frac{(\gamma+s)}{m_{1} m_{0}} M_{1}\left(\int_{\Omega}|\nabla \underline{u}|^{p} d x\right)\left\{\int_{\Omega}|\nabla \phi|^{p-2} \nabla \phi \cdot[\nabla(\phi \varphi)-\varphi \nabla \phi] d x\right\} \\
&=\frac{(\gamma+s)}{m_{1} m_{0}} M_{1}\left(\int_{\Omega}|\nabla \underline{u}|^{p} d x\right)\left\{\int_{\Omega}|\nabla \phi|^{p-2} \nabla \phi \cdot \nabla(\phi \varphi) d x\right\} \\
&-\frac{(\gamma+s)}{m_{1} m_{0}} M_{1}\left(\int_{\Omega}|\nabla \underline{u}|^{p} d x\right)\left\{\int_{\Omega}|\nabla \phi|^{p} \varphi d x\right\} \\
&=\frac{(\gamma+s)}{m_{1} m_{0}} M_{1}\left(\int_{\Omega}|\nabla \underline{u}|^{p} d x\right)\left\{\int_{\Omega} \lambda_{p}|\phi|^{p-2} \phi \cdot(\phi \varphi) d x\right\} \\
&-\frac{(\gamma+s)}{m_{1} m_{0}} M_{1}\left(\int_{\Omega}|\nabla \underline{u}|^{p} d x\right)\left\{\int_{\Omega}|\nabla \phi|^{p} \varphi d x\right\} \\
&= \frac{(\gamma+s)}{m_{1} m_{0}} M_{1}\left(\int_{\Omega}|\nabla \underline{u}|^{p} d x\right) \int_{\Omega}\left(\lambda_{p} \phi^{p}-|\nabla \phi|^{p}\right) \varphi d x \\
& \leq \frac{(\gamma+s)}{m_{0}} \int_{\Omega}\left(\lambda_{p} \phi^{p}-|\nabla \phi|^{p}\right) \varphi d x . \\
&
\end{aligned}
$$


On $\bar{\Omega}_{\delta}$, we have $|\nabla \phi|^{p}-\lambda_{p} \phi^{p} \geq m_{0}$, which implies that

$$
\begin{aligned}
\frac{(\gamma+s)}{m_{0}}\left(\lambda_{p} \phi^{p}-|\nabla \phi|^{p}\right) \leq & -\gamma-s \\
\leq & -\lambda_{1} a_{0} f\left((\gamma+s)^{\frac{1}{p-1}}\right)-\mu_{1} c_{0} h\left((\gamma+s)^{\frac{1}{p-1}}\right) \\
\leq & -\lambda_{1} a_{0} f\left((\gamma+s)^{\frac{1}{p-1}} \frac{p-1}{p}\left(\frac{1}{m_{2} m_{0}}\right)^{\frac{1}{p-1}} \phi^{\frac{p}{p-1}}\right) \\
& -\mu_{1} c_{0} h\left((\gamma+s)^{\frac{1}{p-1}} \frac{p-1}{p}\left(\frac{1}{m_{1} m_{0}}\right)^{\frac{1}{p-1}} \phi^{\frac{p}{p-1}}\right) \\
\leq & \lambda_{1} a(x) f(\underline{v})+\mu_{1} c(x) h(\underline{u}) .
\end{aligned}
$$

Next, on $\Omega \backslash \bar{\Omega}_{\delta}$ we have $\phi \geq r$ for some $r>0$, and therefore by the condition $\left(H_{2}\right)$ and the definition of $\underline{u}, \underline{v}$, it follows that

$$
\begin{aligned}
(\gamma+s)\left(\lambda_{p} \phi^{p}-|\nabla \phi|^{p}\right) \leq & (\gamma+s) \lambda_{p} \\
\leq & \lambda_{1} a_{1} f\left((\gamma+s)^{\frac{1}{p-1}} \frac{p-1}{p}\left(\frac{1}{m_{2} m_{0}}\right)^{\frac{1}{p-1}} r^{\frac{p}{p-1}}\right) \\
& +\mu_{1} c_{1} h\left((\gamma+s)^{\frac{1}{p-1}} \frac{p-1}{p}\left(\frac{1}{m_{1} m_{0}}\right)^{\frac{1}{p-1}} r^{\frac{p}{p-1}}\right) \\
\leq & \lambda_{1} a(x) f(\underline{v})+\mu_{1} c(x) h(\underline{u}),
\end{aligned}
$$

for $\left(\lambda_{1}, \mu_{1}\right) \in[A, B]$.

Relations (2.2) and (2.3) implies that

$$
M_{1}\left(\int_{\Omega \backslash \bar{\Omega}_{\delta}}|\nabla \underline{u}|^{p} d x\right) \int_{\Omega \mid \bar{\Omega}_{\delta}}|\nabla \underline{u}|^{p-2} \nabla \underline{u} \cdot \nabla \varphi d x \leq \int_{\Omega \backslash \bar{\Omega}_{\delta}}\left[\lambda_{1} a(x) f(\underline{v})+\mu_{1} c(x) h(\underline{u})\right] \varphi d x,
$$

for any $\varphi \in W_{0}^{1, p}(\Omega)$ with $\varphi \geq 0$ in $\Omega$. Similarly,

$$
M_{1}\left(\int_{\Omega \backslash \bar{\Omega}_{\delta}}|\nabla \underline{v}|^{p} d x\right) \int_{\Omega \mid \bar{\Omega}_{\delta}}|\nabla \underline{v}|^{p-2} \nabla \underline{v} \cdot \nabla \varphi d x \leq \int_{\Omega \backslash \bar{\Omega}_{\delta}}\left[\lambda_{2} b(x) g(\underline{v})+\mu_{2} d(x) \tau(\underline{u})\right] \varphi d x,
$$

for any $\varphi \in W_{0}^{1, p}(\Omega)$ with $\varphi \geq 0$ in $\Omega$. From (2.4) and (2.5), $(\underline{u}, \underline{v})$ is a subsolution of problem (1.1). Moreover, we have $\underline{u}>0$ and $\underline{v}>0$ in $\Omega$.

Next, we shall construct a supersolution of problem (1.1). For this purpose, let $\phi_{0}$ be the solution of the following problem

$$
\begin{cases}-\Delta_{p} \varphi=1 & \text { in } \Omega \\ \varphi=0, & \text { on } \partial \Omega\end{cases}
$$


Let

$$
\bar{u}:=\frac{C}{R} \phi_{0}, \quad \bar{v}:=\frac{1}{m_{2}^{\frac{1}{p-1}}}[2 g(C)]^{\frac{1}{p-1}} \phi_{0},
$$

where $R=\left\|\phi_{0}\right\|>0$ and $C>0$ is a large positive real number to be chosen later. We shall verify that $(\bar{u}, \bar{v})$ is a supersolution of problem (1.1). To this end, let $\varphi \in W_{0}^{1, p}(\Omega)$ with $\varphi \geq 0$ in $\Omega$, from $\left(H_{1}\right)$, we have

$$
\begin{aligned}
& M_{1}\left(\int_{\Omega}|\nabla \bar{u}|^{p} d x\right) \int_{\Omega}|\nabla \bar{u}|^{p-2} \nabla \bar{u} \cdot \nabla \varphi d x \\
& =M_{1}\left(\int_{\Omega}|\nabla \bar{u}|^{p} d x\right)\left(\frac{C}{R}\right)^{p-1} \int_{\Omega}\left|\nabla \phi_{0}\right|^{p-2} \nabla \phi_{0} \cdot \nabla \varphi d x \\
& =\left(\frac{C}{R}\right)^{p-1} M_{1}\left(\int_{\Omega}|\nabla \bar{u}|^{p} d x\right) \int_{\Omega} \varphi d x \\
& \geq m_{1}\left(\frac{C}{R}\right)^{p-1} \int_{\Omega} \varphi d x .
\end{aligned}
$$

Using the condition $\left(H_{3}\right)$, we can choose the number $C>0$ large enough so that

$$
C^{p-1} \geq \frac{R^{p-1}}{m_{1}}\left(\lambda_{1}\|a\|_{\infty} f\left(\frac{1}{m_{2}^{\frac{1}{p-1}}}[2 g(C)]^{\frac{1}{p-1}} \phi_{0}\right)+\mu_{1}\|c\|_{\infty} h\left(\frac{C}{R}\left\|\phi_{0}\right\|_{\infty}\right)\right)
$$

and therefore

$$
\begin{aligned}
& M_{1}\left(\int_{\Omega}|\nabla \bar{u}|^{p} d x\right) \int_{\Omega}|\nabla \bar{u}|^{p-2} \nabla \bar{u} \cdot \nabla \varphi d x \\
& \geq m_{1}\left(\frac{C}{R}\right)^{p-1} \int_{\Omega} \varphi d x \\
& \geq \int_{\Omega}\left(\lambda_{1}\|a\|_{\infty} f\left(\frac{1}{m_{2}^{\frac{1}{p-1}}}[2 g(C)]^{\frac{1}{p-1}} \phi_{0}\right)+\mu_{1}\|c\|_{\infty} h\left(\frac{C}{R}\left\|\phi_{0}\right\|_{\infty}\right)\right) \varphi d x \\
& \geq \int_{\Omega}\left[\lambda_{1} a(x) f(\bar{v})+\mu_{1} c(x) h(\bar{u})\right] \varphi d x .
\end{aligned}
$$

Next, from the definition of $\bar{v}$, the conditions $\left(H_{1}\right),\left(H_{5}\right),\left(H_{4}\right)$ and the fact that $g$ is increasing, we also deduce that 


$$
\begin{aligned}
& M_{2}\left(\int_{\Omega}|\nabla \bar{v}|^{p} d x\right) \int_{\Omega}|\nabla \bar{v}|^{p-2} \nabla \bar{v} \cdot \nabla \varphi d x \\
& =M_{2}\left(\int_{\Omega}|\nabla \bar{v}|^{p} d x\right) \frac{1}{m_{2}} 2 g(C) \int_{\Omega}\left|\nabla \phi_{0}\right|^{p-2} \nabla \phi_{0} \cdot \nabla \varphi d x \\
& =M_{2}\left(\int_{\Omega}|\nabla \bar{v}|^{p} d x\right) \frac{1}{m_{2}} 2 g(C) \int_{\Omega} \varphi d x \\
& \geq 2 g(C) \int_{\Omega} \varphi d x \\
& \geq \int_{\Omega} 2 g\left(\frac{C}{R} \phi_{0}\right) \varphi d x \\
& =\int_{\Omega}\left[g\left(\frac{C}{R} \phi_{0}\right)+g\left(\frac{C}{R} \phi_{0}\right)\right] \varphi d x \\
& \geq \int_{\Omega}\left[\lambda_{2}\|b\|_{\infty} g(\bar{u})+\tau(\bar{v})\right] \varphi d x \\
& \geq \int_{\Omega}\left[\lambda_{2}\|b\|_{\infty} g(\bar{u})+\mu_{2}\|d\|_{\infty} \tau(\bar{v})\right] \varphi d x \\
& \geq \int_{\Omega}\left[\lambda_{2} b(x) g(\bar{u})+\mu_{2} d(x) \tau(\bar{v})\right] \varphi d x .
\end{aligned}
$$

From (2.8) and (2.9), $(\bar{u}, \bar{v})$ is a supersolution of problem (1.1) with $\underline{u} \leq \bar{u}$ and $\underline{v} \leq \bar{v}$ for $C>0$ large.

In order to obtain a weak solution of problem (1.1) we shall use the arguments by Azzouz and Bensedik [4]. For this purpose, we define a sequence $\left\{\left(u_{n}, v_{n}\right)\right\} \subset W_{0}^{1, p}\left(\Omega, \mathbb{R}^{2}\right)$ as follows: $u_{0}:=\bar{u}, v_{0}:=\bar{v}$ and $\left(u_{n}, v_{n}\right)$ is the unique solution of the system

$$
\left\{\begin{array}{lc}
-M_{1}\left(\int_{\Omega}\left|\nabla u_{n}\right|^{p} d x\right) \Delta_{p} u_{n}=\lambda_{1} a(x) f\left(v_{n-1}\right)+\mu_{1} c(x) h\left(u_{n-1}\right) & \text { in } \Omega, \\
-M_{2}\left(\int_{\Omega}\left|\nabla v_{n}\right|^{p} d x\right) \Delta_{p} v_{n}=\lambda_{2} b(x) g\left(u_{n-1}\right)+\mu_{2} d(x) \tau\left(v_{n-1}\right) & \text { in } \Omega, \\
u_{n}=v_{n}=0 & \text { on } \partial \Omega .
\end{array}\right.
$$

System (2.10) is $\left(M_{1}, M_{2}\right)$-linear in the sense that, if $\left(u_{n-1}, v_{n-1}\right) \in W_{0}^{1, p}\left(\Omega, \mathbb{R}^{2}\right)$ is given, the right hand sides of (2.10) is independent of $u_{n}, v_{n}$. Then we deduce from a result in [3] that system (2.10) has a unique solution $\left(u_{n}, v_{n}\right) \in W_{0}^{1, p}\left(\Omega, \mathbb{R}^{2}\right)$.

Using (2.10) and the fact that $\left(u_{0}, v_{0}\right)$ is a supersolution of (1.1) we have

$$
\begin{aligned}
& -M_{1}\left(\int_{\Omega}\left|\nabla u_{0}\right|^{p} d x\right) \Delta_{p} u_{0} \geq \lambda_{1} a(x) f\left(v_{0}\right)+\mu_{1} c(x) h\left(u_{0}\right)=-M_{1}\left(\int_{\Omega}\left|\nabla u_{1}\right|^{p} d x\right) \Delta_{p} u_{1}, \\
& -M_{2}\left(\int_{\Omega}\left|\nabla v_{0}\right|^{p} d x\right) \Delta_{p} v_{0} \geq \lambda_{2} b(x) g\left(u_{0}\right)+\mu_{2} d(x) \tau\left(v_{0}\right)=-M_{2}\left(\int_{\Omega}\left|\nabla v_{1}\right|^{p} d x\right) \Delta_{p} v_{1},
\end{aligned}
$$


and by Proposition 1.1, $u_{0} \geq u_{1}$ and $v_{0} \geq v_{1}$. Also, since $u_{0} \geq \underline{u}, v_{0} \geq \underline{v}$, then by the monotonicity of $f, h, \tau, g$ one has

$$
\begin{aligned}
-M_{2}\left(\int_{\Omega}\left|\nabla u_{1}\right|^{p} d x\right) \Delta_{p} u_{1} & =\lambda_{1} a(x) f\left(v_{0}\right)+\mu_{1} c(x) h\left(u_{0}\right) \\
& \geq \lambda_{1} a(x) f(\underline{v})+\mu_{1} c(x) h(\underline{u}) \\
& \geq-M_{2}\left(\int_{\Omega}|\nabla \underline{u}|^{p} d x\right) \Delta_{p} \underline{u}, \\
-M_{2}\left(\int_{\Omega}\left|\nabla v_{1}\right|^{p} d x\right) \Delta_{p} v_{1} & =\lambda_{2} b(x) g\left(u_{0}\right)+\mu_{2} d(x) \tau\left(v_{0}\right) \\
& \geq \lambda_{2} b(x) g(\underline{u})+\mu_{2} d(x) \tau(\underline{v}) \\
& \geq-M_{2}\left(\int_{\Omega}|\nabla \underline{v}|^{p} d x\right) \Delta_{p} \underline{v},
\end{aligned}
$$

from which, according to Proposition 1.1, $u_{1} \geq \underline{u}, v_{1} \geq \underline{v}$. For $u_{2}, v_{2}$ we write

$$
\begin{aligned}
-M_{1}\left(\int_{\Omega}\left|\nabla u_{1}\right|^{p} d x\right) \Delta_{p} u_{1} & =\lambda_{1} a(x) f\left(v_{0}\right)+\mu_{1} c(x) h\left(u_{0}\right) \\
& \geq \lambda_{1} a(x) f\left(v_{1}\right)+\mu_{1} c(x) h\left(u_{1}\right) \\
& =-M_{1}\left(\int_{\Omega}\left|\nabla u_{2}\right|^{p} d x\right) \Delta_{p} u_{2}, \\
-M_{2}\left(\int_{\Omega}\left|\nabla v_{1}\right|^{p} d x\right) \Delta_{p} v_{1} & =\lambda_{2} b(x) g\left(u_{0}\right)+\mu_{2} d(x) \tau\left(v_{0}\right) \\
& \geq \lambda_{2} b(x) g\left(u_{1}\right)+\mu_{2} d(x) \tau\left(v_{1}\right) \\
& =-M_{2}\left(\int_{\Omega}\left|\nabla v_{2}\right|^{p} d x\right) \Delta_{p} v_{2}
\end{aligned}
$$

and then $u_{1} \geq u_{2}, v_{1} \geq v_{2}$. Similarly, $u_{2} \geq \underline{u}$ and $v_{2} \geq \underline{v}$ because

$$
\begin{aligned}
-M_{1}\left(\int_{\Omega}\left|\nabla u_{2}\right|^{p} d x\right) \Delta_{p} u_{2} & =\lambda_{1} a(x) f\left(v_{1}\right)+\mu_{1} c(x) h\left(u_{1}\right) \\
& \geq \lambda_{1} a(x) f(\underline{v})+\mu_{1} c(x) h(\underline{u}) \\
& \geq-M_{1}\left(\int_{\Omega}|\nabla \underline{u}|^{p} d x\right) \Delta_{p} \underline{u}
\end{aligned}
$$




$$
\begin{aligned}
-M_{2}\left(\int_{\Omega}\left|\nabla v_{2}\right|^{p} d x\right) \Delta_{p} v_{2} & =\lambda_{2} b(x) g\left(u_{1}\right)+\mu_{2} d(x) \tau\left(v_{1}\right) \\
& \geq \lambda_{2} b(x) g(\underline{u})+\mu_{2} d(x) \tau(\underline{v}) \\
& \geq-M_{2}\left(\int_{\Omega}|\nabla \underline{v}|^{p} d x\right) \Delta_{p} \underline{v} .
\end{aligned}
$$

Repeating this argument we get a bounded monotone sequence $\left\{\left(u_{n}, v_{n}\right)\right\} \subset W_{0}^{1, p}\left(\Omega, \mathbb{R}^{2}\right)$ satisfying

$$
\begin{aligned}
& \bar{u}=u_{0} \geq u_{1} \geq u_{2} \geq \cdots \geq u_{n} \geq \cdots \geq \underline{u}>0, \\
& \bar{v}=v_{0} \geq v_{1} \geq v_{2} \geq \cdots \geq v_{n} \geq \cdots \geq \underline{v}>0 .
\end{aligned}
$$

Using the continuity of the functions $f, g, h, \tau$, the definition of the sequences $\left\{u_{n}\right\},\left\{v_{n}\right\}$, there exist four constants $C_{1}, C_{2}, C_{3}, C_{4}>0$ independent of $n$ such that

$$
\left|f\left(v_{n-1}\right)\right| \leq C_{1}, \quad\left|g\left(u_{n-1}\right)\right| \leq C_{2}, \quad\left|h\left(u_{n-1}\right)\right| \leq C_{3}, \quad\left|\tau\left(v_{n-1}\right)\right| \leq C_{4} \quad \text { for all } n .
$$

From (2.12), multiplying the first equation of (2.10) by $u_{n}$, integrating, using the Hölder inequality and the Sobolev embedding we can show that

$$
\begin{aligned}
m_{1} \int_{\Omega}\left|\nabla u_{n}\right|^{p} d x & \leq M_{1}\left(\int_{\Omega}\left|\nabla u_{n}\right|^{p} d x\right) \int_{\Omega}\left|\nabla u_{n}\right|^{p} d x \\
& =\int_{\Omega}\left(\lambda_{1} a(x) f\left(v_{n-1}\right)+\mu_{1} c(x) h\left(u_{n-1}\right)\right) u_{n} d x \\
& \leq \int_{\Omega}\left(\lambda_{1}\|a\|_{\infty}\left|f\left(v_{n-1}\right)\right|+\mu_{1}\|c\|_{\infty}\left|h\left(u_{n-1}\right)\right|\right)\left|u_{n}\right| d x \\
& \leq C \int_{\Omega}\left|u_{n}\right| d x \\
& \leq C^{\prime}\left\|u_{n}\right\|,
\end{aligned}
$$

or

$$
\left\|u_{n}\right\| \leq C^{\prime}, \quad \forall n,
$$

where $C^{\prime}>0$ is a constant independent of $n$. Similarly, there exists $C^{\prime \prime}>0$ independent of $n$ such that

$$
\left\|v_{n}\right\| \leq C^{\prime \prime}, \quad \forall n \text {. }
$$

From (2.14), (2.15) we infer that $\left\{\left(u_{n}, v_{n}\right)\right\}$ has a subsequence which converges in $L^{p}\left(\Omega, \mathbb{R}^{2}\right)$ to a limit $(u, v)$ with the properties $u \geq \underline{u}>0$ and $v \geq \underline{v}>0$. Being monotone, $\left\{\left(u_{n}, v_{n}\right)\right\}$ converges itself to $(u, v)$. Now, lettting $n \rightarrow \infty$ in (2.10), we deduce that $(u, v)$ is a positive solution of system (1.1). This completes the proof.

\section{References}

[1] G.A. Afrouzi, N.T. Chung And S. Shakeri, Positive solutions for a semipositone problem involving nonlocal operator, Rend. Semin. Mat. Univ. Padova 132 (2014), 25-32. 
[2] G.A. Afrouzi, N.T. Chung And S. Shakeri, Existence and non-existence results for nonlocal elliptic systems via sub-supersolution method, Funkcial. Ekvac. 59 (2016), 303-313.

[3] C.O. Alves And F.J.S.A. CORRÊA, On existence of solutions for a class of problem involving a nonlinear operator, Comm. Appl. Nonlinear Anal. 8 (2001), 43-56.

[4] N. Azzouz AND A. BensediK, Existence results for an elliptic equation of Kirchhoff-type with changing sign data, Funkcial. Ekvac. 55 (2012), 55-66.

[5] M. Chipot And B. Lovat, Some remarks on non local elliptic and parabolic problems, Nonlinear Anal. 30 (1997), 4619-4627.

[6] F.J.S.A. CorrêA AND G.M. Figueiredo, On an elliptic equation of $p$-Kirchhoff type via variational methods, Bull. Aust. Math. Soc., 74 (2006), 263-277.

[7] F.J.S.A. CorrêA AND G.M. Figueiredo, On a $p$-Kirchhoff equation via Krasnoselskii's genus, Appl. Math. Lett. 22 (2009), 819-822.

[8] G. Dai, Three solutions for a nonlocal Dirichlet boundary value problem involving the $p(x)$-Laplacian, Appl. Anal. 92 (2013), 191-210.

[9] G. Dai and R. Ma, Solutions for a $p(x)$-Kirchhoff type equation with Neumann boundary data, Nonlinear Anal. Real World Appl. 12 (2011), 2666-2680.

[10] D.D. Hai AND R. Shivaji, An existence result on positive solutions for a class of $p$-Laplacian systems, Nonlinear Anal. 56 (2004), 1007-1010.

[11] G. KIRCHHOFF, Mechanik, Teubner, Leipzig, Germany, 1883.

[12] T.F. MA, Remarks on an elliptic equation of Kirchhoff type, Nonlinear Anal. 63 (2005), 1967-1977.

[13] K. Perera And Z. Zhang, Nontrivial solutions of Kirchhoff-type problems via the Yang index, J. Differential Equations, 221 (2006), 246-255.

[14] B. RICCERI, On an elliptic Kirchhoff-type problem depending on two parameters, J. Global Optim. 46 2010, 543-549.

[15] J.-J. SUn AND C.-L. TANG, Existence and multiplicity of solutions for Kirchhoff type equations, Nonlinear Anal. 74 (2011), 1212-1222.

[16] Z. Zhang And K. Perera, Sign changing solutions of Kirchhoff type problems via invariant sets of descent flow, J. Math. Anal. Appl. 317 (2006), 456-463. 